\title{
Dynamical mass determination for the very low mass stars LHS 1070 B and C
}

\author{
Ch. Leinert ${ }^{1}$, H. Jahreiß ${ }^{2}$, J. Woitas ${ }^{1}$, S. Zucker ${ }^{3}$, T. Mazeh ${ }^{3}$, A. Eckart ${ }^{4}$, and R. Köhler ${ }^{1, \star}$ \\ 1 Max-Planck-Institut für Astronomie, Königstuhl 17, 69117 Heidelberg, Germany \\ 2 Astronomisches Rechen-Institut, Mönchhofstr. 12-14, 69120 Heidelberg, Germany \\ 3 Wise Observatory, Tel Aviv University, Tel Aviv 69978, Israel \\ 4 Physikalisches Institut der Universität Köln, Germany
}

Received 12 October 2000 / Accepted 29 November 2000

\begin{abstract}
We followed the relative orbit of the close pair of very late-type stars in the nearby triple system LHS 1070 during the past seven years. These observations are used to derive the astrometric elements of the orbit. Using the period of $16.1 \pm 1.4$ years and assuming the distance of $7.39 \mathrm{pc}$ given by van Altena (1995) to be exactly correct, the combined mass of components $\mathrm{B}$ and $\mathrm{C}$ is $0.138 \pm 0.003 M_{\odot}$. Otherwise, this mass scales with the third power of the distance, which presently is not known to better than $9 \%$. The dynamical mass would equal the combined mass of $0.161 \pm 0.002 M_{\odot}$ derived from the theoretical mass-luminosity relation of Baraffe et al. (1998) and Chabrier et al. (2000) for a $5 \%$ larger distance of 7.78 pc. A critical comparison with the theoretical relations therefore has to await more precise parallax determinations for this comparatively faint system with visual magnitude $V=15.3$. The wide orbit of component A around the center of mass of components B and $\mathrm{C}$ cannot yet be determined from our measurements. However, we can show that it is probably co-planar with the close pair orbit and should have a period in the range $60-1000$ years.
\end{abstract}

Key words. low-mass stars - binaries - mass-luminosity relation

\section{Introduction}

LHS 1070 (other common name GJ 2005) is a nearby 15th magnitude M5V star with high proper motion located in the south galactic pole region. With its velocity component perpendicular to the galactic plane of $36 \pm 3 \mathrm{~km} \mathrm{~s}^{-1}$ (Basri \& Marcy 1995), it is considered a member of the old disk population with a probable age of several Gigayears - a typical late M dwarf.

In 1993 Leinert et al. (1994) discovered a pair of cool, very low-mass stars as close companions to LHS 1070 , turning the system into a nearby triple system. This gave the system a special status, because it now held the promise of dynamical mass determination at the lower end of the main sequence. Subsequent photometry and spectroscopy with the Hubble Space Telescope (Leinert et al. 2000) confirmed that companions B and C, with spectral types of $\mathrm{M} 8.5 \mathrm{~V}$ and $\mathrm{M} 9-9.5 \mathrm{~V}$, respectively, may be the least luminous stars within $10 \mathrm{pc}$ from the sun, and indeed should have masses close to the main sequence cutoff. Absolute visual magnitudes - depending on the

Send offprint requests to: Ch. Leinert,

e-mail: leinert@mpia-hd.mpg.de

* Based on data obtained from the $3.6 \mathrm{~m}$ and NTT ESO telescopes on La Silla, from the Calar Alto $3.5 \mathrm{~m}$ telescope, and from the Hubble Space Telescope WFPC and NICMOS instruments. assumed distance - of $M_{V}=15.6-16.0,19.0-19.3$ and 19.4-19.7 were found for components A, B and C, respectively (Leinert et al. 2000). Comparison with the theoretical mass-luminosity relations of Baraffe et al. (1998) and Chabrier et al. (2000) led Leinert et al. (2000) to estimated masses of 0.079-0.080 $M_{\odot}$ for component $\mathrm{C}$ and 0.080-0.083 $M_{\odot}$ for component $\mathrm{B}$. The estimated ranges include the dependence on the assumed distance, which is only weak, since the mass-luminosity relation is very steep in this mass range.

A direct dynamical determination to support or correct the theoretical relationships for these very low-mass stars would help to better understand the lower end of the main sequence and the transition between low-mass stars and brown dwarfs. The triple system LHS 1070, containing two very low-mass components in a comparatively shortperiod orbit and having in addition a primary as an astrometric reference, if needed, was unique in this respect. Only very recently Martín et al. (2000) discovered that Gliese 569 is actually another triple system with properties similar to those of LHS 1070, with an even shorter period of $\approx 3$ years. In this paper we present the first reliable orbit determination for the close pair of the LHS 1070 system, derived from observations that followed the relative orbit over seven years. We then discuss the resulting dynamical masses of the components. 
Table 1. Observed positions of LHS 1070 component C with respect to component B

\begin{tabular}{|c|c|c|c|c|c|c|c|c|c|}
\hline Date & $\begin{array}{l}\rho \\
\left({ }^{\prime \prime}\right)\end{array}$ & $\begin{array}{l}\theta \\
\left({ }^{\circ}\right)\end{array}$ & Telescope & Instrument & $\begin{array}{c}\text { Pixel size } \\
\left({ }^{\prime \prime}\right)\end{array}$ & $\begin{array}{l}X_{\mathrm{c}} \\
\left({ }^{\prime \prime}\right)\end{array}$ & $\begin{array}{r}\Delta X \\
\left({ }^{\prime \prime}\right)\end{array}$ & $\begin{array}{r}Y_{\mathrm{C}} \\
\left({ }^{\prime \prime}\right)\end{array}$ & $\begin{array}{r}\Delta Y \\
\left.{ }^{\prime \prime}\right)\end{array}$ \\
\hline July 29, 1993 & $0.266 \pm 0.005$ & $328.5 \pm 0.7$ & ESO NTT & SHARP I & 0.050 & 0.228 & -0.001 & -0.135 & -0.004 \\
\hline May 1, 1994 & $0.341 \pm 0.0$ & $346.9 \pm 0.6$ & ESO NTT & SHAl & 049 & 0.332 & 0.001 & -0.076 & -0.001 \\
\hline Sep. 15, 1994 & $375 \pm 0$ & $352.7 \pm 0.61$ & Calar Alto $3.5 \mathrm{~m}$ & Blue MAGIC & 0.071 & 0.373 & -0.001 & -0.045 & -0.003 \\
\hline Sep. 24, 1994 & $0.382 \pm 0.0$ & $353.9 \pm 0.67$ & ESO $3.6 \mathrm{~m}$ & ADONIS/SHARP II & 050 & 0.375 & 0.005 & -0.043 & 0.002 \\
\hline Jan. 17, 1995 & $0.400 \pm 0.008$ & $356.1 \pm 0.4$ & ESO $3.6 \mathrm{~m}$ & IS/SHA & 0.050 & 0.403 & -0.004 & -0.016 & -0.012 \\
\hline July 9, 1995 & $0.439 \pm 0.004$ & $3.2 \pm 0.1$ & ESO NTT & SHARP I & 0.049 & 0.434 & 0.004 & 0.026 & -0.001 \\
\hline July 14,1995 & $0.436 \pm 0$ & $3.7 \pm 0.2$ & HST & WFPC2 & 0.046 & 0.435 & 0.000 & 0.027 & 0.001 \\
\hline Jan. 1 & $0.459 \pm$ & $9.0 \pm 0.3$ & ESO $3.6 \mathrm{~m}$ & $\mathrm{ADC}$ & 0.050 & 0.453 & 0.001 & 0.069 & 0.002 \\
\hline Aug. 22, 1996 & $0.465 \pm 0$ & $14.9 \pm 0.1$ & ESO $3.6 \mathrm{~m}$ & $\mathrm{ADO}$ & 0.050 & 0.455 & -0.005 & 0.118 & 0.002 \\
\hline ep. 27,1996 & $0.468 \pm 0.004$ & $15.8 \pm 0.1$ & Calar Alto $3.5 \mathrm{~m}$ & AGIC & 0.071 & 0.453 & -0.003 & 0.125 & 0.002 \\
\hline uly 15,1997 & $0.458 \pm 0.006$ & $23.4 \pm 0.6$ & ESO NTT & SHARP I & 0.050 & 0.418 & 0.003 & 0.179 & 0.003 \\
\hline 25,1997 & $0.450 \pm 0$ & $25.4 \pm 1.0$ & ESO $3.6 \mathrm{~m}$ & $\mathrm{ADC}$ & 0.050 & 0.410 & -0.003 & 0.185 & 0.008 \\
\hline Nov. 17,1997 & $0.439 \pm 0.008$ & $26.5 \pm 0.45$ & Calar Alto $3.5 \mathrm{~m}$ & AGIC & 0.071 & 0.392 & 0.001 & 0.197 & -0.001 \\
\hline Jan. 2, 1998 & $0.432 \pm 0.0$ & $28.0 \pm 0.45$ & HST & $\mathrm{NIC}$ & 0.043 & 0.381 & 0.001 & 0.203 & -0.001 \\
\hline May 7, 1998 & $0.408 \pm 0.008$ & $32.3 \pm 0.73$ & ESO NTT & SHA & 0.049 & 0.347 & -0.002 & 0.218 & 0.000 \\
\hline Oct. 10, 1998 & $0.377 \pm 0.019$ & $38.2 \pm 0.5$ & Calar Alto $3.5 \mathrm{~m}$ & A-CASS & 0.095 & 0.298 & -0.002 & 0.232 & 0.001 \\
\hline June 18, 1999 & $0.318 \pm 0.002$ & $49.4 \pm 0.4$ & ESO NTT & SHA & 0.049 & 0.205 & 0.002 & 0.242 & -0.001 \\
\hline Aug. 3, 1999 & $0.303 \pm 0.007$ & $52.1 \pm 0.3$ & Calar Alto 3. & OMEGA-CA & 0.095 & 0.187 & -0.001 & 0.243 & -0.004 \\
\hline Sep. 1, 1999 & $0.292 \pm 0.008$ & $54.6 \pm 1.0$ & Calar Alto $3.5 \mathrm{~m}$ & OMEGA-CASS & 0.095 & 0.175 & -0.006 & 0.243 & -0.005 \\
\hline Nov. 23, 1999 & $0.279 \pm 0.0$ & $60.1 \pm 0.9$ & Calar Alto 3 & OMEGA-CASS & 0.095 & 0.140 & -0.001 & 0.242 & 0.001 \\
\hline & & & & & & & & 0.233 & 0.001 \\
\hline June 20,2000 & $0.237 \pm 0.003$ & $80.8 \pm 0.7$ & ESO NTT & SHARP I & 0.050 & 0.048 & -0.010 & 0.231 & 0.002 \\
\hline
\end{tabular}

$X_{\mathrm{c}}, Y_{\mathrm{c}}$ are the positions predicted from the orbital fit, $\Delta X$ and $\Delta Y$ the differences observed - predicted.

LHS 1070 appears even to be a quadruple system. Henry et al. (1999) detected a fourth component, D, in close orbit around component A, with $M_{V}=17.8$. The existence of this fourth component affects only marginally the results and conclusions of our study.

\section{Observations}

Since components B and C of the close pair in LHS 1070 are only separated by $00^{\prime \prime} 2-0 . \prime 5$, we observed them with high spatial resolution techniques, usually near-infrared speckle interferometry or adaptive optics observations. For consistency and ease of handling we also reduced the adaptive optics data with the reduction procedures developed for speckle observations (Köhler et al. 2000). Journal of observations and measured relative orbital positions are given in Table 1. This is a heterogeneous data set obtained on different telescopes with different instruments in different observing techniques over a longer period of time. Therefore, standardization of the obtained positional results is of first importance. We found it most practical to refer all our observations to the geometry of the inner Trapezium cluster obtained with the same instrument during the same campaign. For the Trapezium stars, accurate astrometric observations are available (McCaughrean \& Stauffer 1994), and we derived plate scale and orientation of the instrument from such Trapezium observations. For the campaign of July 1995, Menten et al. (1997) derived plate scale and orientation for the same instrument from a comparison of near-infrared and VLA radio measurements of sources in the Galactic Center with
$\mathrm{SiO}$ maser emission. Our results agreed with theirs within $10^{-3}$ in plate scale and $0.15^{\circ}$ in detector orientation. At several subsequent observing runs we have simultaneously observed the Galactic Center and the Trapezium cluster core and have determined pixel scale and detector orientation both with respect to McCaughrean \& Stauffer's (1994) and Menten et al.'s (1997) astrometries, establishing a confirmed relationship between these two reference frames. This allowed us, in the few cases when Trapezium observations were not available, to resort to observations of the galactic center for calibration of the measured relative position. Typically, the observations were performed in $K$ band $(2.2 \mu \mathrm{m})$ with pixel sizes of 0. ' 05 , but observations with a pixel size of $00^{\prime \prime} 1$ also occurred. The determination of pixel scale usually is accurate to $\pm 0.5 \%$, and the orientation to $\pm 0.2^{\circ}$, which translates into an uncertainty of $\approx 2$ milliarcsec (mas) for the separation of the pair BC.

\section{Results}

The relative orbital positions obtained for the close pair BC during the period 1993-2000 are summarized in Table 1. The average uncertainty of individual positions - including calibration uncertainty - is $\approx 5$ mas, which sounds optimistic for measurements with a diffraction limit of $0^{\prime \prime} 13$. However, it is our experience that the separation of two point sources can be measured to a precision of a couple of milliarcsec with the near-infrared speckle technique, and this is confirmed by the residuals of the orbital fit to the data, which is shown in Fig. 1. 


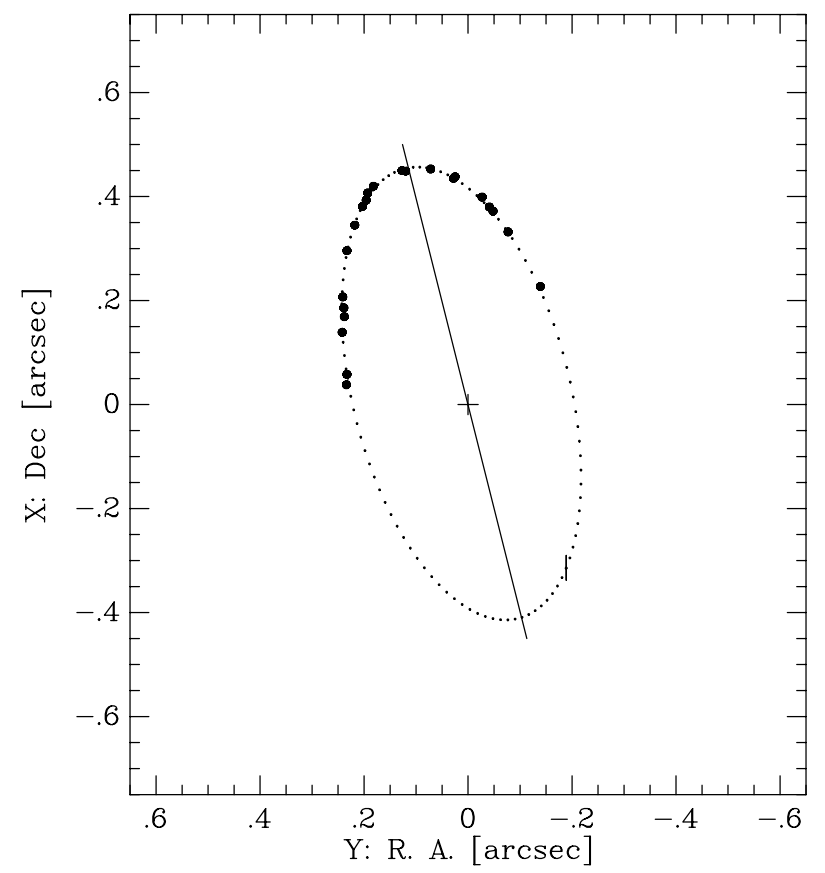

Fig. 1. The relative orbit of LHS $1070 \mathrm{C}$ around component $\mathrm{B}$, assumed to be fixed at the origin. The observations are represented by full dots $(\bullet)$. The result of the orbital fit is shown by the small dots, giving orbital positions in temporal steps of $1 / 100$ of the orbital period. The differences observed minus computed are always smaller than the dot size. The line of nodes is indicated by a full line, and the periastron by a small upright dash. The position of the periastron is not well determined in this nearly circular orbit. It could even fall to the left of the line of nodes

The parameters of the fitted Keplerian orbit are shown in Table 2. These parameters were determined by differential correction of the rectangular coordinates as described in Heintz (1978). A satisfactory fit could be obtained only now with the available 112 degrees coverage of the orbit. Of course, the direction of periastron remains rather undetermined due to the very small eccentricity. The errors of the orbital parameters in Table 2 were determined by the Jacknife method according to Mosteller \& Tukey (1977), and are in excellent accordance with the rms errors resulting from the least squares fit for the three directly fitted parameters $P, e, T_{\circ}$, which amount to $\pm 1.4 \mathrm{yrs}, \pm 0.061$, and \pm 0.7 , respectively. As can be seen from Table 1 , differences between observed and computed orbit larger than \pm 0.005 arcsec are rather exceptional, the average value of these residuals being 4.1 mas. Table 3 presents the ephemeris for the remainder of the present orbit.

At first glance, there is a discrepancy between the apparently well determined mutual gravitational interaction of the two components (see Fig. 1) and the size of the errors for the parameters of the relative orbit. These errors of $6.5 \%$ in semimajor axis and $8.9 \%$ in period would, after application of Kepler's third law, lead to an error
Table 2. Parameters of the orbital fit and resulting masses

\begin{tabular}{lcl}
\hline Parameter & Value & Error or Comment \\
\hline$a\left(^{\prime \prime}\right)$ & 0.446 & \pm 0.029 \\
$e$ & 0.064 & \pm 0.063 \\
$i^{\circ}$ & 62.1 & \pm 1.5 \\
$\Omega^{\circ}$ & 14.1 & \pm 0.6 \\
$\omega^{\circ}$ & 213 & \pm 26 \\
$P(\mathrm{yr})$ & 16.1 & \pm 1.4 \\
$T_{\circ}$ & 1990.2 & \pm 0.7 \\
& & \\
$\pi\left({ }^{\prime \prime}\right)$ & 0.1353 & \pm 0.0121 \\
$d(\mathrm{pc})$ & 7.5 & assumed \\
$a(\mathrm{AU})$ & $3.35 \times d_{7.5}$ & $\pm 0.22 \times d_{7.5}$ \\
$M_{\mathrm{B}}+M_{\mathrm{C}}\left(M_{\odot}\right)$ & $0.144 \times d_{7.5}^{3}$ & $\pm 0.003 \times d_{7.5}^{3}$ \\
\hline
\end{tabular}

Note: $d_{7.5}=d_{\mathrm{LHS} 1070} /(7.5 \mathrm{pc})$.

Table 3. Ephemeris for LHS 1070BC

\begin{tabular}{lrr}
\hline date & $\theta\left(^{\circ}\right)$ & $\rho\left({ }^{\prime \prime}\right)$ \\
\hline 2001.0 & 100.1 & 0.216 \\
2002.0 & 141.2 & 0.250 \\
2003.0 & 166.5 & 0.331 \\
2004.0 & 182.0 & 0.400 \\
2005.0 & 194.1 & 0.422 \\
2006.0 & 206.5 & 0.388 \\
2007.0 & 223.7 & 0.307 \\
2008.0 & 255.2 & 0.220 \\
2009.0 & 304.0 & 0.213 \\
2010.0 & 338.0 & 0.297 \\
\hline
\end{tabular}

in the mass determination of $\pm 26 \%$, even when neglecting the $9 \%$ error in parallax. However, these formal errors are not uncorrelated. For example, a longer period could fit the data, which cover a substantial part of the orbit, only together with a larger semimajor axis. When deriving the mass of the system these changes largely cancel. This could be seen when we repeated the orbital fit with a parameter fixed at a non-optimal value. In short, apart from the uncertainties in the parallax, the error on the combined mass of components $\mathrm{B}$ and $\mathrm{C}$ is $\pm 0.003 M_{\odot}$.

At present, the error on the published parallax of 0 ". $1353 \pm 0$.'0121 (van Altena et al. 1995) is uncomfortably large, but there are no particular reasons why no more precise parallax measurement could be obtained on this object, and we assume that this will happen during the coming years. Therefore we decided to treat the distance to LHS 1070 as a variable and allow for it by the parameter $d_{7.5}=d_{\text {LHS1070 }} /(7.5 \mathrm{pc})$, where $7.5 \mathrm{pc}$ is the nearest round value to the published parallax. The combined mass of the components $\mathrm{B}$ and $\mathrm{C}$ then results as $(0.144 \pm 0.003) \cdot d_{7.5}^{3} M_{\odot}$. Using the published parallax with its given error, the resulting combined mass would turn out to be $0.138 \pm 0.037 M_{\odot}$. We prefer to present our results in Table 2 in the first notation, which emphasizes the potential of the results by showing that in principle the relevant accuracy corresponding to a few percent in mass has been reached. 


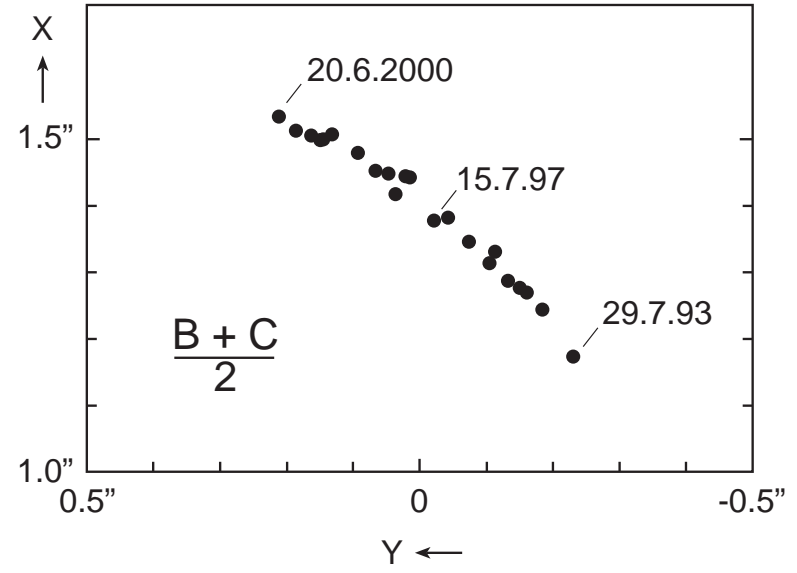

Fig. 2. Orbital motion of the center of mass of components B and $\mathrm{C}$ with respect to the main component $\mathrm{A}$ of the system LHS 1070. For this plot, equal masses have been assumed for $\mathrm{B}$ and $\mathrm{C}$, the components of the close pair. The zero point for the axes is in component A, North is up, east is left

Table 4. Motion of the geometrical center of components B and $\mathrm{C}$ with respect to component $\mathrm{A}$

\begin{tabular}{rcc}
\hline $\begin{array}{r}\text { Date of } \\
\text { observation }\end{array}$ & $\begin{array}{c}X\left(^{\prime \prime}\right) \\
\text { (north positive) }\end{array}$ & $\begin{array}{c}Y\left({ }^{\prime \prime}\right) \\
\text { (east positive) }\end{array}$ \\
\hline July 29, 1993 & 1.174 & -0.231 \\
May 1, 1994 & 1.241 & -0.184 \\
Sep. 15, 1994 & 1.269 & -0.161 \\
Sep. 24, 1994 & 1.277 & -0.151 \\
Jan. 17, 1995 & 1.288 & -0.132 \\
July 9, 1995 & 1.331 & -0.113 \\
July 14, 1995 & 1.314 & -0.104 \\
Jan. 16, 1996 & 1.346 & -0.074 \\
Aug. 22, 1996 & 1.381 & -0.042 \\
Sep. 27, 1996 & 1.378 & -0.021 \\
July 15, 1997 & 1.443 & 0.015 \\
Aug. 25, 1997 & 1.445 & 0.021 \\
Nov. 17, 1997 & 1.418 & 0.036 \\
Jan. 2, 1998 & 1.449 & 0.047 \\
May 7, 1998 & 1.453 & 0.066 \\
Oct. 10, 1998 & 1.480 & 0.093 \\
June 18, 1999 & 1.508 & 0.132 \\
Aug. 3, 1999 & 1.500 & 0.145 \\
Sep. 1, 1999 & 1.499 & 0.149 \\
Nov. 23, 1999 & 1.506 & 0.162 \\
June 4, 2000 & 1.501 & 0.187 \\
June 20, 2000 & 1.534 & 0.211 \\
\hline
\end{tabular}

\section{The wide orbit}

We define the wide orbit by the motion of the distant component A around the center of mass of the close pair BC. In Table 4 and Fig. 2 we show our astrometric measurements of the geometrical center of the BC system relative to component $\mathrm{A}$. These measurements cover an arc of $19^{\circ}$ of the wide orbit, with a resulting areal constant of $0.045 \square^{\prime \prime} /$ year. Note, however, that the geometrical center is not exactly the center of mass of the BC pair, although the difference should be less than 5 mas if the masses of $\mathrm{B}$ and $\mathrm{C}$ are as similar as concluded from optical

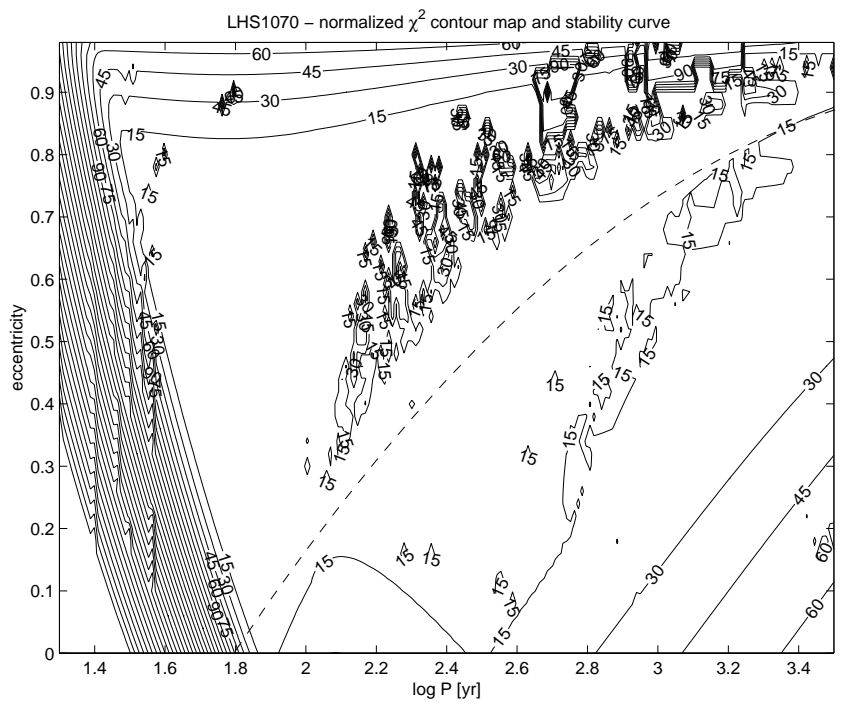

Fig. 3. Allowed period-eccentricity range for the wide orbit. The solid lines present the contour map of the normalized $\chi^{2}$ (mas) resulting from fits to the astrometric data of Table 4. Large part of the log $P-e$ plane yields fits with normalized $\chi^{2}$ smaller than 15 mas. The dashed line represents the theoretical stability limit predicted on the basis of the known period of the close orbit. Only points below this line are allowed

photometry (Leinert et al. 2000). Furthermore, the existence of the additional fourth component of the system, D, induces perturbation of the actual and observed position of A, (see Sect. 4.3), inserting a further unknown contribution into our measurements. With these uncertainties, it is surprising that we, nevertheless, succeeded to derive some interesting conclusions about the wide orbit and the relative inclination of the two orbits.

\subsection{Period and eccentricity}

To be able to derive some properties of the wide orbit in spite of the present short coverage we searched for any combination of period and eccentricity that is allowed by the astrometric data. To do that we solved for all the other parameters of the wide orbit for any given combination of period and eccentricity, and derived the corresponding $\chi^{2}$ statistics. The result is presented in Fig. 3, where we plotted a contour map of the minimum normalized $\chi^{2}$ obtained for the possible realisations of the wide orbit as a function of $\log P$ and $e$. The normalized $\chi^{2}$ was calculated as

$\sqrt{\frac{1}{m-n} \sum\left(\frac{\mathrm{O}_{i}-\mathrm{C}_{i}}{1 \mathrm{mas}}\right)^{2}}$

where $\mathrm{O}_{i}$ and $\mathrm{C}_{i}$ are the observed and calculated position of the $i$ th measurement, $m$ is the number of measurements and $n$ is the number of free parameters, 6 in our case. Usually, when solving visual binaries, there are 7 free parameters. In this case, the total mass estimate, together with the parallax and Kepler's Harmonic Law, force a relation between the period and the semi-major axis. 


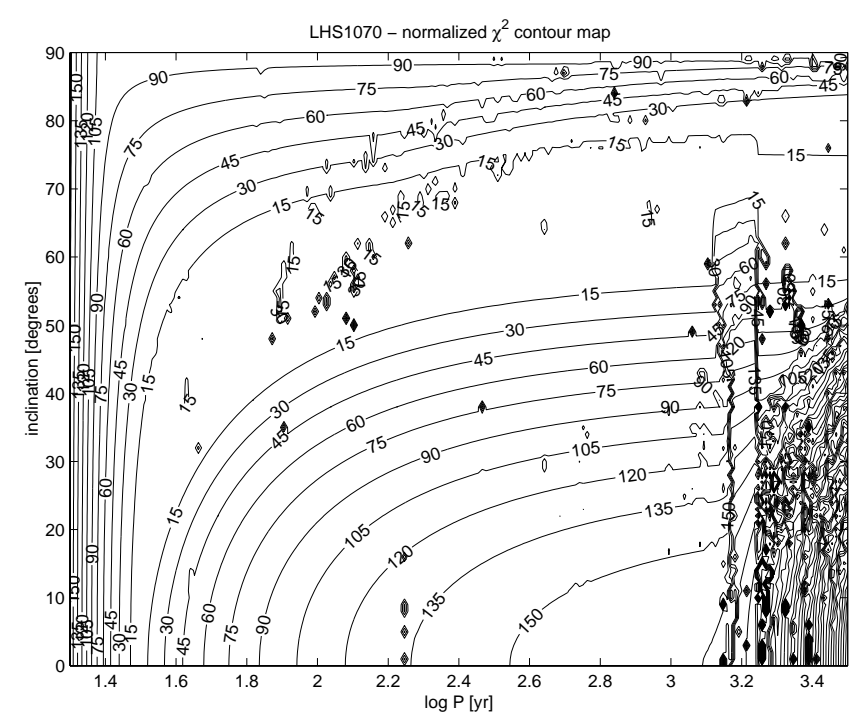

Fig. 4. Most probable values of period and inclination for the wide orbit derived from fits to the astrometric data of Table 4. The best values lie in the "valley" of normalized $\chi^{2}<15$ mas, and for a wide range of periods correspond to $i \approx 60^{\circ}$

The above expression gives the mean deviation of the calculated orbit from the measurements in units of mas.

Most of the period-eccentricity plane is below the 15 mas level. In principle, the period and eccentricity of the wide orbit could be anywhere in this section.

We can further constrain the permitted section of the period-eccentricity plane by requiring that the system should be dynamically stable. We approximate the system to an hierarchical triple system, where the distant companion is A, ignoring the probable existence of component D. In general, when the distant companion gets too close to the close binary, tidal interaction exerted on the $\mathrm{BC}$ pair leads to disruption of the system. Therefore for each wide-orbit period there is a maximum stable eccentricity, above which the triple system is unstable (Harrington 1992; Eggleton \& Kiseleva 1995). This eccentricity is plotted in Fig. 3 as a dashed line. The line was derived by three-body numerical simulations, using Severe Aarseth (Aarseth \& Zare 1974) numerical code, assuming coplanarity of the two orbits and equal masses for the three components. In general, we found that when the minimum distance of the distant companion is larger than the maximum separation of the close pair by a factor of 3.7 or more the system is stable. Some configurations with a factor of only 3.5 show stability over a few millions close orbits. The exact borderline between the stable and the unstable part of the diagram has still to be studied in detail, a study which is outside the scope of this paper.

Even the coarse borderline plotted in Fig. 3 well constrains the possible combinations of period and eccentricity for the wide orbit. The figure suggests that the period could be in the range of 60-1000 years, with an eccentricity that varies accordingly from almost circular to quite large values $(e=0.7)$.

\subsection{Coplanarity}

We performed a similar study in the period-inclination plane, the results of which are plotted in Fig. 4. The figure shows a "valley" of the normalized $\chi^{2}$ values which starts at $\log P$ of about 1.5 and inclination $0^{\circ}$ and goes to higher inclinations almost without a change in the period. The "valley" then turns to higher periods with only very moderate inclination increase. Now, when we impose on this figure the constraint derived from the period-eccentricity analysis, namely that the period could be only in the range of $60-1000$ years, we get that the inclination is at about $50-60^{\circ}$. We find this result extremely intriguing, as the inclination of the close pair is $62^{\circ}$. The probability that this similarity happened by chance is very small, and we therefore suggest, instead, that this attests to the nearcoplanarity of the two orbits. If this is true, the longitude of the nodes, $\Omega$, of the two orbits should be with similar values as well. Unfortunately, it seems that the present data do not constrain well enough the values of $\Omega$ and the coplanarity remains to be checked in the future when a larger part of the wide orbit is observed.

\subsection{Influence of component $D$ on the wide orbit}

The presence of the fourth component D (Henry et al. 1999) orbiting component A in close orbit of about one year (T. Henry, private communication) complicates the situation. Strictly speaking, the wide orbit now has to be defined as the motion of the center of mass of the close pair AD around the center of mass of the pair BC.

With its visual brightness of $V=17.8$ (Henry et al. 1999), component $\mathrm{D}$ is roughly half way between the brightnesses of components A and C. Assuming a similar relation at $2.2 \mu \mathrm{m}$, the brightness ratio at $\mathrm{K}$ between components $\mathrm{A}$ and $\mathrm{D}$ would be 2 , and the semimajor axis of the center of light motion only about $1 / 6$ of the semimajor axis for the motion of the pair AD. The resulting disturbance on the wide orbit then would be at most 10 mas, difficult to detect with the present data set. However, once definite predictions exist for the orbit and mass of component $\mathrm{D}$, a search for the reflex signature in the wide orbit of the system LHS 1070 appears promising.

\section{Masses}

The combined mass for components B and C of LHS 1070, assuming the published parallax value to be accurate, is $0.138 \pm 0.003 M_{\odot}$. Based on their similar brightness, with, e.g., $\Delta V=0.4 \mathrm{mag}$ (Leinert et al. 2000), one would expect their masses to agree within 3\% (Baraffe et al. 1998; Chabrier et al. 2000). This would mean that both components should have masses very close to $0.070 M_{\odot}$, below the hydrogen burning limit, and therefore would classify as massive brown dwarfs. This, however, is not compatible with the spectral analysis given in Leinert et al. (2000), which sees components $\mathrm{B}$ and $\mathrm{C}$ as very low mass stars. We conclude that the published parallax value probably gives 


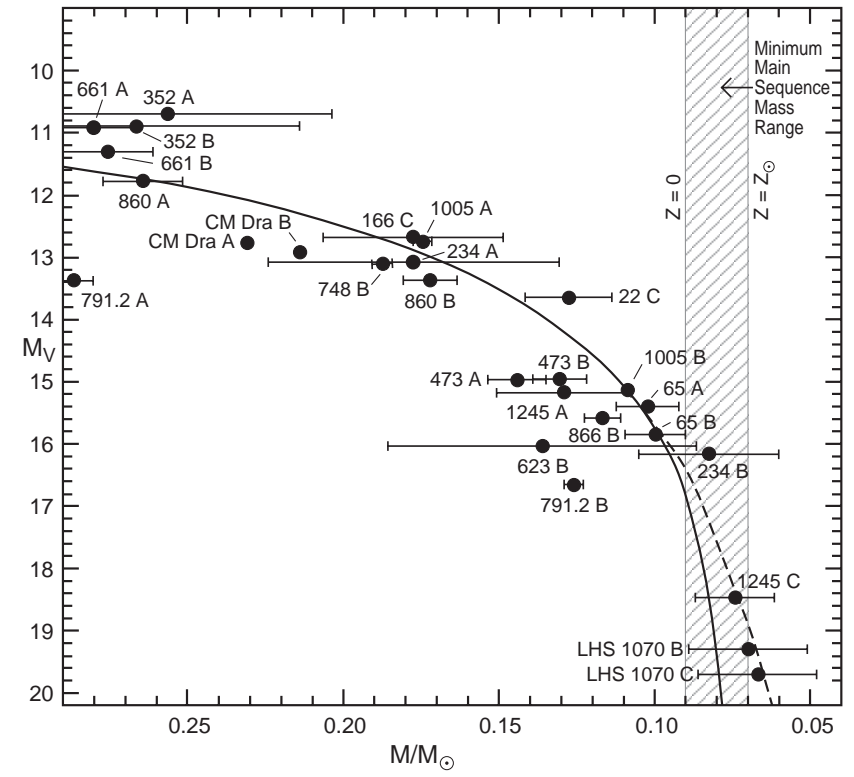

Fig. 5. Mass-luminosity diagram with the components B and C of LHS 1070 included. For this plot, it was assumed that component $\mathrm{C}$ is less massive than component $\mathrm{B}$ by $3 \%$, as would be expected from the difference in brightness. The other data points refer to the eclipsing binary CM Dra, to HST Fine Guidance Sensor observations (like Gliese 65 A and 65 B), to a combination of radial velocity and adaptive optics observations (like Gliese 866 B) or to speckle interferometric work. The theoretical mass-luminosity relationships according to Baraffe et al. (1998) and Chabrier et al. (2000) are given as solid line for an age of $5 \mathrm{Gyr}$, while the broken line shows the relation for an age of 0.5 Gyr. The error bars for LHS 1070 B and C reflect the uncertainty in parallax. With accurate parallax, the $1 \sigma$ error would correspond to the diameter of the dot symbol. The data for this figure, apart from LHS 1070, were kindly provided by Todd Henry

too small a distance, and we note that an increase of the distance to LHS 1070 by $5 \%$ to the value of 7.78 pc would just result in the same combined mass of $0.161 \pm 0.002 M_{\odot}$ as found from the theoretical mass-luminosity relations of Baraffe et al. (1998) and Chabrier et al. (2000).

The $9 \%$ error on the published parallax, which corresponds to a $30 \%$ error in combined mass, at present is the main obstacle to useful application of our results. Figure 5 gives the present status of the mass-luminosity relation for very low mass stars, where we have included components $\mathrm{B}$ and C of LHS 1070 with the values and errors resulting from the published parallax of van Altena et al. (1995). We see that the mass uncertainty is larger than, e.g., the difference between the mass-luminosity relations for $0.5 \mathrm{Gyr}$ and 5 Gyr. To exploit the discriminating power of our measurements, we simply have to wait for more precise parallax determinations on this nearby $V=15.3$ mag system. Such observations certainly are feasible and hopefully will become available during the next few years. Then, LHS 1070 B and C probably will be the two lowest-mass $M$ dwarfs for which accurate dynamical masses have been determined.

\section{Conclusion}

We have determined dynamical masses for the supposedly $0.08 M_{\odot}$ objects LHS $1070 \mathrm{~B}$ and $\mathrm{C}$ by following their relative orbit. We have shown that the data allow, in principle, to determine the masses to a few percent accuracy, yielding a good definition of the mass-luminosity relation at the lower end of the main sequence. However, the present uncertainty in parallax is too large to give our results the discriminating power they deserve. This problem should and probably will disappear during the coming years when high precision parallaxes should become available also for this nearby triple or most probably quadruple system.

Acknowledgements. We thank our colleagues who were friendly enough to perform an orbital position measurement for us or even donated the necessary time for it from their campaign, and thus helped us to overcome the difficulty in getting the many separated short observations always at reasonable weather conditions: David Barrado, Jean-Luc Beuzit, Patrice Bouchet, Jochen Eislöffel, Tom Herbst, Mark McCaughrean, Hans Zinnecker. We also thank Thomas Ott for providing images of the Galactic Center for plate scale calibrations, and William Hartkopf for providing ephemerides of visual binary stars for the same purpose. The work of T. M. and S. Z. was supported by the Israeli Science Foundation.

\section{References}

Aarseth, S. J., \& Zare, K. 1974, Celes. Mech., 10, 185

Altena, W. F., van, Lee, J. T., \& Hoffleit, E. D. 1995, The General Catalogue of Trigonometric Stellar Parallaxes, 4th edition (Yale University Obs., New Haven)

Baraffe, I., Chabrier, G., Allard, F., \& Hauschildt, P. H. 1998, A\&A, 337, 403

Basri, G., \& Marcy, G. W. 1995, AJ, 109, 762

Chabrier, G., Baraffe, I., Allard, F., \& Hauschildt, P. 2000, ApJ, 542, 464

Eggleton, P., \& Kiseleva, L. 1995, ApJ, 455, 640

Harrington, R. S. 1992, in ASP Conf. Ser. 32, Complementary approaches to double and multiple star research, Proc. IAU Colloquium No. 135, ed. H. A. McAlister, \& W. Hartkopf, San Francisco, 212

Heintz, W. D. 1978, Double Stars (D. Reidel Publ. Comp., Dordrecht)

Henry, T. J., Franz, O. G., Wasserman, L. H., et al. 1999, ApJ, 512,864

Köhler, R., Kunkel, M., Leinert, Ch., \& Zinnecker, H. 2000, A\&A, 356, 541

Leinert, Ch., Weitzel, N., Richichi, A., Eckart, A., \& TacconiGarman, L. E. 1994, A\&A, 291, L47

Leinert, Ch., Allard, F., Richichi, A., \& Hauschildt, P. H. 2000, A\&A, 353, 691

McCaughrean, M. J., \& Stauffer, J. R. 1994, AJ, 108, 1382

Martín, E. L., Koresko, C. D., Kulkarni, S. R., Lane, B. F., \& Wizinowitch, P. L. 2000, ApJ, 529, L37

Menten, K. M., Reid, M. J., Eckart, A., \& Genzel, R. 1997, ApJ, 475, L111

Mosteller, F., \& Tukey, J. W. 1977, Data Analysis and Regression (Addison-Wesley Publ. Comp.) 